\title{
Optimal revascularization in diabetes after the FREEDOM trial: Were the controversies finally settled?
}

\author{
Alexander Tenenbaum ${ }^{1,2,3}$, Enrique Z. Fisman ${ }^{2,3}$ \\ ${ }^{1}$ Cardiac Rehabilitation Institute, Sheba Medical Center, Tel-Hashomer, Israel \\ ${ }^{2}$ Sackler Faculty of Medicine, Tel-Aviv University, Tel-Aviv, Israel \\ ${ }^{3}$ Cardiovascular Diabetology Research Foundation, Holon, Israel
}

\begin{abstract}
The prevalence of diabetes mellitus (DM) is growing worldwide. Prothrombotic and proinflammatory states, in adjunct to endothelial dysfunction and metabolic disorders, such as hyperglycemia, dyslipidemia, obesity, insulin resistance, and oxidative stress, are key features of the accelerated atherosclerotic progression observed in patients with DM. Moreover, drug-eluting stents (DES) thrombosis rate was higher in DM than in non-DM patients and DM itself was identified as an independent predictor of stent thrombosis, particularly due to the impaired response to dual antiplatelet therapy. The accumulating data even before the FREEDOM trial provided strong evidence that in patients with DM and complex coronary artery disease, coronary artery bypass grafting (CABG) was superior to percutaneous coronary intervention (PCI) which was based on the first-generation DES. The FREEDOM trial enrolled 1900 patients with DM and multivessel coronary artery disease treated with CABG surgery or PCI with the first-generation DES. The patients were followed for a median 3.8 years; $C A B G$ was superior to PCI as it significantly reduced rates of death and myocardial infarction (MI), with a higher rate of stroke. The benefit of $C A B G$ was driven by differences in rates of both $M I(p<0.001)$ and death from any cause $(p=0.049)$. Following the FREEDOM results, patients with DM ought to be informed before coronary angiography about the potential survival benefit from $C A B G$ for the treatment of a complex disease. However, it should be noticed that the second generation DES were associated with better outcomes compared to the first-generation DES. New stent designs are continually being developed, with the aim of further improving the clinical efficacy and the safety profile of these devices. Therefore, although the results of the FREEDOM trial clearly demonstrated that CABG was superior to PCI in DM, a comparative analysis of the new incoming stents warrants further investigation. (Cardiol J 2013; 20, 4: 331-336)
\end{abstract}

Key words: coronary artery disease, diabetes mellitus

\section{FREEDOM trial: A brief synopsis} of the official results

Sponsored by the National Heart, Lung, and Blood Institute, the Future Revascularization Evaluation in Patients with Diabetes Mellitus: Optimal Management of Multivessel Disease (FREEDOM) trial enrolled patients with diabetes mellitus (DM) and multivessel coronary artery disease (CAD) in a treatment with a coronary artery bypass grafting $(\mathrm{CABG})$ surgery or a percutaneous coronary intervention (PCI) with sirolimus-eluting stents (SES) and paclitaxel-eluting stents (PES). SES and PES were used exclusively in $51 \%$ and $43 \%$ of pa-

Address for correspondence: Prof. Alexander Tenenbaum, MD, PhD, Director of Research, Cardiac Rehabilitation Institute, Sheba Medical Center, 52621 Tel-Hashomer, Israel, tel: 9723 5302578, e-mail: altenen@post.tau.ac.il 
tients, respectively, in the PCI group who actually underwent PCI [1]. Newer-generation stents could be used as long as they were FDA-approved. Dual antiplatelet therapy was recommended for at least 12 months. From April 2005 through April 2010, a total of 32,966 patients were screened and only 3309 were found to be trial-eligible. Out of them, $1900(57.4 \%)$ patients provided written informed consent and underwent randomization at 140 international centers. The patients were followed for a minimum of 2 years (a median among survivors, 3.8 years).

The patients' mean age was $63.1 \pm 9.1$ years, $29 \%$ were women, and $83 \%$ had 3 -vessel disease. The primary outcome (a composite of death from any cause, nonfatal myocardial infarction [MI], and a nonfatal stroke) occurred more frequently in the PCI group ( $p=0.005)$, with 5 -year rates of $26.6 \%$ in the PCI group and $18.7 \%$ in the CABG group. The benefit of CABG was driven by differences in rates of both MI $(\mathrm{p}<0.001)$ and death from any cause $(p=0.049)$. The stroke was more frequent in the CABG group, with 5 -year rates of $2.4 \%$ in the $\mathrm{PCI}$ group and $5.2 \%$ in the CABG group $(\mathrm{p}=0.03)$. Most of the strokes (87\%) were ischemic strokes and $13 \%$ were hemorrhagic. So, in patients with diabetes and advanced CAD, CABG was superior to $\mathrm{PCI}$ in that it significantly reduced rates of death and MI, with a higher rate of stroke.

\section{Diabetes mellitus: Why is cardiovascular disease so common and revascularization so challenging?}

The prevalence of DM is growing worldwide. DM itself is the major cause of accelerated atherogenesis leading to atherothrombosis and diabetic patients have a $2-4$ times higher risk of cardiovascular disease and an up to 3 times increased risk of mortality compared to the non-DM population. In the developed countries, up to a quarter of all coronary revascularization procedures - either CABG or PCI - may involve DM patients [2-4].

Prothrombotic and proinflammatory states, in adjunct to endothelial dysfunction and metabolic disorders, such as hyperglycemia, dyslipidemia, obesity, insulin resistance, and oxidative stress, are key features of the accelerated atherosclerotic progression observed in patients with DM $[5,6]$. The prothrombotic status is the consequence of multiple conditions, including increased platelet reactivity; increased levels of procoagulant agents such as fibrinogen, tissue factor, von Willebrand factor, platelet factor 4, factor VII; decreased con- centrations of endogenous anticoagulants including protein $\mathrm{C}$ and antithrombin III; and impaired endogenous fibrinolysis secondary to elevated levels of plasminogen activator inhibitor-1 [7, 8].

Multiple mechanisms contribute to increased platelet aggregation in DM patients. First, hyperglycemia may induce the expression of the surface adhesion molecule P-selectin, the glycation of platelet surface proteins with a consequent membrane fluidity decrease and a platelet adhesion increase, the activation of protein kinase $\mathrm{C}$, and may exert a direct osmotic effect [9-12]. Hyperglycemia may also promote atherothrombosis via oxidation of amino groups, formation of advanced glycation end-products, endothelial dysfunction, subendothelial cellular proliferation, and increased matrix expression $[12,13]$. Other abnormalities that contribute to the enhanced platelet adhesion and activation in DM include the increased expression of the platelet receptor glycoprotein (GP) IIb/IIIa, up-regulation of platelet $\mathrm{P} 2 \mathrm{Y}_{12}$ receptor signalling, increased platelet turnover, and enhanced oxidative stress. In addition, insulin resistance may increase intracellular calcium concentration and impair the response to nitric oxide [9-13].

\section{Diabetes mellitus: Data previous to FREEDOM}

The main limitation of bare metal stents (BMS)-based PCI, and particularly in DM patients, is restenosis. A meta-analysis of 6 BMS trials, including 1,166 DM and 5,070 non-DM patients, detected a restenosis rate of $37 \%$ in DM patients and identified DM as an independent predictor of restenosis (odds ratio [OR] 1.3) [14]. Even in the drug-eluting stents (DES) era, DM patients undergoing PCI have worse outcomes compared with non-DM individuals. Particularly, diabetic women who have undergone PCI are a high-risk group warranting special attention in treatment strategies [15]. Furthermore, DM patients - especially those treated with insulin - had higher mortality as well as stent thrombosis and target vessel revascularization (TVR) rates.

A network meta-analysis of 35 randomized trials comparing DES with BMS and including 3,852 DM patients showed that the use of DES, while not affecting overall mortality or MI rates, was associated with a $60-70 \%$ relative risk reduction in target lesion revascularization (TLR) depending on the type of stent used [16]. A beneficial effect of DES vs. BMS in DM was confirmed in a large prospective registry of consecutive patients 
allowing for a comparison of 2 propensity-matched cohorts of 1476 patients each undergoing DES or BMS implantation. The 3-year risk-adjusted mortality, MI, and TVR rates in the DES vs. BMS propensity matched cohorts were 17.5 vs. $20.7 \%$ $(\mathrm{p}=0.02), 13.8 \%$ vs. $16.9 \%(\mathrm{p}=0.02)$, and $18.4 \%$ vs. $23.7 \%$ ( $\mathrm{p}<0.001)$, respectively [17].

Most of the large-scale clinical investigations showed that the DES thrombosis rate was higher in $\mathrm{DM}$ than in non-DM patients and on several occasions DM was identified as an independent predictor of stent thrombosis. This observation, together with the marked reduction in this complication associated with potent platelet inhibitors such as prasugrel or ticagrelor, supports the notion that the prothrombotic state and the impaired response to dual antiplatelet therapy observed in DM patients are of an overwhelming importance [18-21].

The accumulating evidence of PCI vs. CABG was pooled in a meta-analysis before the FREEDOM trial. Ten randomized trials with a total of 7,812 patients were included to compare the effectiveness of CABG with PCI [22]. During a median follow-up of 5.9 years, mortality in diabetics after CABG $(\mathrm{n}=615)$ was $23 \%$ and $29 \%$ after PCI $(\mathrm{n}=618)$ (hazard ratio $[\mathrm{HR}]=0.70,95 \%$ confidence interval [CI] 0.56-0.87). These data provided strong evidence that survival is substantially higher after CABG than PCI for the treatment of multivessel disease. These findings were not only a result of the inclusion of the BARI trial since the trend in survival remains after exclusion of that trial. Six early randomized controlled trials (with a total of 950 diabetics) compared CABG and PCI [23]. In diabetic patients with multivessel CAD a survival advantage and fewer repeat revascularization procedures were demonstrated with an initial surgical revascularization. However, due to advances in medical therapy, PCI technology, and surgical techniques, this conclusion was questioned in the present era. More recent clinical trials should compare new revascularization strategies specifically in diabetic patients to define the optimal management strategy.

The first prospective randomized trial dedicated to evaluate the safety and efficacy of PCI compared with those of CABG in patients with diabetes is the Coronary Artery Revascularization in Diabetes trial (CARDia), which presented their 1-year follow-up results [24]. A total of 510 diabetic patients with multivessel or complex single-vessel $\mathrm{CAD}$ were randomized and treated with either CABG or PCI using initially BMS (31\%) and later SES $(69 \%)$. The primary outcome, defined as a composite of all-cause mortality, MI, and stroke, was $10.5 \%$ in the CABG group and $13.0 \%$ in the PCI group $(\mathrm{p}=0.39)$. The secondary outcome included the addition of repeat revascularization to the primary outcome events and did show a difference between the two groups: $11.3 \%$ for CABG vs. $19.3 \%$ in PCI $(\mathrm{p}=0.02)$. When the patients who underwent $\mathrm{CABG}$ were compared with the subset of patients who received DES, the secondary outcome rates were comparable $(12.9 \%$ and $18.0 \%$; HR $=1.41,95 \%$ CI $0.82-2.42$ ).

Before FREEDOM, the only data from a randomized trial that exclusively used DES comes from the SYNTAX trial. It was the first to compare a DES, in this case a PES, to CABG as the treatment of left main disease (isolated or in addition to 1-, 2-, or 3-vessel disease) or 3-vessel disease in a randomized controlled trial. A recent subgroup analysis showed 3-year outcomes of the trial by the treatment strategy and the subgroup [25]. Among this group, 452 patients were medically treated for diabetes with either hypoglycemic agents $(59.7 \%)$ or insulin $(40.3 \%)$. Patients treated with a diet alone were considered as nondiabetic. The diagnosis was made at the time of enrollment. Data of complete antidiabetic medication use and new diagnoses of diabetics after enrollment were unfortunately not available.

Clinical endpoints were major adverse cardiac and cerebrovascular events (MACCE) that included all-cause death, stroke, MI, and repeat revascularization. Patient demographics and baseline lesion characteristics between the randomized $\mathrm{CABG}$ and PES diabetic subgroups were well matched with the exception of an increased incidence of high triglycerides $(\geq 150 \mathrm{mg} / \mathrm{dL}$ ) in CABG compared with PES ( $47.1 \%$ vs. $37.0 \%$, $\mathrm{p}=0.04)$ and increased incidence of elevated blood pressure $\geq 130 / 85 \mathrm{~mm} \mathrm{Hg}$ in PES (65.2\% vs. $74.5 \%, \mathrm{p}=0.03)$ [26].

The 3-year outcome showed a significant increased rate of MACCE in the diabetic PES arm $(37.0 \%$ vs. $22.9 \%, p=0.002)$, mainly caused by the increased rate of repeat revascularization ( $28.0 \%$ vs. $12.9 \%, p<0.001)$. The overall rate of the composite of death/stroke/MI was not significantly different between the PES and CABG arms $(16.3 \%$ vs. $14.0 \%$, respectively; $p=0.527)$. Both in the non-DM and the DM subgroup, there were numeric increases in $\mathrm{MI}$ in the PES arm compared with the CABG arm. In the non-DM subgroup, this was a significant difference $(7.5 \%$ vs. $3.2 \%$, $\mathrm{p} \leq 0.001$ ), while in the DM subgroup this did not reach the significance ( $5.8 \%$ vs. $4.8 \%, \mathrm{p}=0.633$ ). 
Before randomization, the angiographic lesion complexity of coronary arteries was graded with the SYNTAX score [27]. There were similar results of the composite safety endpoint (death/stroke/ /MI) and/or repeat revascularization in the PES and CABG groups in the lowest SYNTAX score tertile (score 0-22), representing the lowest lesion complexity. However, as well in the medium (SYNTAX score 23-32) as in the high (SYNTAX score $\geq 33$ ) groups, MACCE was significantly higher in the PES arm compared with the CABG arm: $21.0 \%$ vs. $36.2 \%, \mathrm{p}=0.04$ for the intermediate and $18.5 \%$ vs. $45.9 \%, \mathrm{p}<0.001$ for the highest SYNTAX score, respectively. Furthermore, Hannan et al. [28] have shown that for patients with multivessel disease, CABG is associated with lower mortality rates than the treatment with DES. Therefore the data before FREEDOM show that in patients with diabetes and complex CAD, CABG was superior to $\mathrm{PCI}$ which was based on the first-generation DES. These results are consistent with findings in the general population; almost 1 in 7 PCI patients requires readmission within 30 days of a hospital discharge and approximately $50 \%$ of all readmitted $\mathrm{PCI}$ patients resulted in a repeat revascularization procedure, accompanied by a significantly higher all-cause 30-day mortality rate [29].

\section{Diabetes mellitus: Are all DES created equal?}

After the FREEDOM trial there is an opinion [30] that the superiority of CABG over PCI on hard outcomes remains similar whether PCI is performed without stents, with BMS, or with DES. In the FREEDOM trial SES and PES (both are the first-generation DES) were used as the devices of the same effectiveness. However, a meta-analysis of 5 head-to-head studies dedicated to DM patients $(\mathrm{n}=1173)$ demonstrated that the sirolimus-eluting Cypher (Cordis) stent was more effective than the paclitaxel-eluting Taxus stent (Boston Scientific) with respect to TLR (5.1\% vs. $11.4 \%$; OR 0.41 , $\mathrm{p}<0.001)$ and angiographic binary restenosis (5.6\% vs. $16.4 \%$; OR $0.30, \mathrm{p}<0.001$ ) [31].

Moreover, the second generation DES (e.g. everolimus-eluting) were associated with better safety outcomes compared to the first-generation DES in patients with DM: diabetics have lower rates of definite stent thrombosis and all-cause mortality with second-generation everolimus-eluting stents (EES) rather than first-generation DES [32]. Left main coronary artery stenting was also shown to be feasible and safe with excellent immediate and mid-term results [33], but since only 13.8 of the study population were diabetics, these promising results should not necessarily be extrapolated to the whole diabetic population.

In the SPIRIT V diabetic study EES was superior to PES for in-stent late loss at 9 months $(0.19 \mathrm{~mm}$ vs. $0.39 \mathrm{~mm}$, respectively; $\mathrm{P}$ (superiority $)=$ $=0.0001)$. However, the composite rate of death, MI, and TVR was the same in the 2 groups at 1 year (16.3\% vs. $16.4 \%)$. The authors concluded that in this prospective, randomized trial in a high-risk group of diabetic patients, implantation of EES compared with PES resulted in significantly better inhibition of intimal hyperplasia with a comparable safety outcome [34]. The EES compared with PES provided significant improvements in clinical safety and efficacy outcomes. The absolute benefit provided by EES vs. PES appears to be proportional to the complexity of coronary disease [35]. In the experimental model, PES exhibited greater neointimal area, increased inflammation, greater medial necrosis, and persistent fibrin compared to EES [36].

Patients from the Swedish Coronary Angiography and Angioplasty Registry (SCAAR) with EES had fewer adverse events than those with PES (HR 1.33) and significantly fewer than with SES (HR 1.99) [32]. This was partially due to less stent thrombosis with EES. HRs for PES and SES were 1.74 and 2.87, respectively. Mortality was also lower with EES, with corresponding HRs of 1.69 and 2.02 with PES and SES, respectively: an advantage compatible with CABG in the FREEDOM trial.

The apparent benefits of EES might reflect the improvements in stent design of this second-generation device. Furthermore, the newer, recently introduced, modern-design rapamycin-analogs or PES with durable bio-absorbable or polymer-free platforms might theoretically represent valid alternatives in diabetic patients.

From a clinical-practice point of view, many interventionalists are able to identify low-risk and high-risk patients and as a result are able to direct them to the most appropriate revascularization treatment. For this reason, some of registry data have shown that clinical events were similar in diabetic patients undergoing CABG surgery or PCI $[37,38]$. On an individual patient level, there are factors such as the risk of stroke, frailty, renal function, pulmonary function, patient preference, operator experience, and other variables that go into making an individualized patient decision in the "real world" practice. What happened with a diabetic patient after emergency PCI of the culprit 
lesion during acute coronary syndrome? Should residual lesions be treated by CABG? It should be particularly pointed that of nearly 33,000 patients who were screened for the trial only 1-tenth were eligible for the inclusion, of whom just 1,900 finally provided informed consent [1]!

Finally, "You can’t stop progress", implying that we are unable to control development of the new technology [39]. New stent designs are continually being developed, with the aim of further improving the clinical efficacy and the safety profile of these devices [40]. In the near future it will be impossible again to identify differences between CABG and PCI based on the newest stents without the requirement of a new randomized trial.

\section{Conclusions}

The results of the FREEDOM trial clearly demonstrated that in patients with diabetes and multivessel CAD, CABG was superior to $\mathrm{PCI}$ which was based on the first-generation DES. CABG significantly reduced rates of death and MI, but was associated with a higher rate of stroke. Obviously, patients with diabetes ought to be informed before coronary angiography about the potential survival benefit from CABG for the treatment of multivessel disease. In any case, it is important to pinpoint that the comparative analysis between CABG and the new PCI technologies based on the recently developed and the upcoming stents warrants further investigation and future revascularization evaluations.

\section{Acknowledgements}

This work was supported in part by the Cardiovascular Diabetology Research Foundation (RA 58-040-684-1), Holon, Israel.

Conflict of interest: none declared

\section{References}

1. Farkouh ME, Domanski M, Sleeper LA et al. Strategies for Multivessel Revascularization in Patients with Diabetes. N Engl J Med, 2012 [Epub ahead of print].

2. Preis SR, Hwang SJ, Coady S et al. Trends in all-cause and cardiovascular disease mortality among women and men with and without diabetes mellitus in the Framingham Heart Study, 1950 to 2005. Circulation, 2009; 119: 1728-1735.

3. Groot MW, Head SJ, Bogers AJ, Kappetein AP. Coronary revascularization in diabetic patients. A focus on the 3-year SYNTAX trial outcomes. Herz, 2012; 37: 281-286.

4. Fox CS, Coady S, Sorlie PD et al. Increasing cardiovascular disease burden due to diabetes mellitus: the Framingham Heart Study. Circulation, 2007; 115: 1544-1550.
5. Creager MA, Lüscher TF, Cosentino F, Beckman JA. Diabetes and vascular isease: pathophysiology, clinical consequences, and medical therapy: Part I. Circulation, 2003; 108: 1527-1532.

6. Biondi-Zoccai GG, Abbate A, Liuzzo G, Biasucci LM. Atherothrombosis, inflammation, and diabetes. J Am Coll Cardiol, 2003; 41: 1071-1077.

7. An X, Yu D, Zhang R et al. Insulin resistance predicts progression of de novo atherosclerotic plaques in patients with coronary heart disease: a one-year follow-up study. Cardiovasc Diabetol, 2012; 11: 71.

8. Ferreiro JL, Angiolillo DJ. Challenges and perspectives of antiplatelet therapy in patients with diabetes mellitus and coronary artery disease. Curr Pharm Des, 2012; 18: 5273-9523.

9. Roffi M, Angiolillo DJ, Kappetein AP. Current concepts on coronary revascularization in diabetic patients. Eur Heart J, 2011; 32: 2748-2757.

10. Bierhaus A, Hofmann MA, Ziegler R, Nawroth PP. AGEs and their interaction with AGE-receptors in vascular disease and diabetes mellitus. I. The AGE concept. Cardiovasc Res, 1998; 37: 586-600.

11. Kassaian SE, Goodarzynejad H, Boroumand MA et al. Glycosylated hemoglobin (HbA1c) levels and clinical outcomes in diabetic patients following coronary artery stenting. Cardiovasc Diabetol, 2012; $11: 82$

12. Ferreiro JL, Gómez-Hospital JA, Angiolillo DJ. Platelet abnormalities in diabetes mellitus. Diab Vasc Dis Res, 2010; 7: 251-259.

13. Aronson D, Rayfield EJ. How hyperglycemia promotes atherosclerosis: molecular mechanisms. Cardiovasc Diabetol, 2002; 1: 1 .

14. Gilbert J, Raboud J, Zinman B. Meta-analysis of the effect of diabetes on restenosis rates among patients receiving coronary angioplasty stenting. Diabetes Care, 2004; 27: 990-994.

15. Blöndal M, Ainla T, Marandi T, Baburin A, Eha J. Sex-specific outcomes of diabetic patients with acute myocardial infarction who have undergone percutaneous coronary intervention: a register linkage study. Cardiovasc Diabetol, 2012; 11: 96.

16. Stettler C, Allemann S, Wandel S et al. Drug eluting and bare metal stents in people with and without diabetes: collaborative network meta-analysis. BMJ, 2008; 337: a1331.

17. Garg P, Normand SL, Silbaugh TS, Wolf RE et al. Drug-eluting or bare-metal stenting in patients with diabetes mellitus: Results from the Massachusetts Data Analysis Center Registry. Circulation, 2008; 118: 2277-2285.

18. Saucedo JF. Antiplatelet therapy for patients with diabetes mellitus and acute coronary syndrome. Prim Care Diabetes, 2012; 6: 167-177.

19. James S, Angiolillo DJ, Cornel JH et al. Ticagrelor vs. clopidogrel in patients with acute coronary syndromes and diabetes: A substudy from the PLATelet inhibition and patient Outcomes (PLATO) trial. Eur Heart J, 2010; 31: 3006-3016.

20. Angiolillo DJ. The evolution of antiplatelet therapy in the treatment of acute coronary syndromes: From aspirin to the present day. Drugs, 2012; 72: 2087-2116.

21. Wiviott SD, Braunwald E, Angiolillo DJ et al. Greater clinical benefit of more intensive oral antiplatelet therapy with prasugrel in patients with diabetes mellitus in the trial to assess improvement in therapeutic outcomes by optimizing platelet inhibition with prasugrel: Thrombolysis in Myocardial Infarction 38. Circulation, 2008; 118: 1626-1636.

22. Hlatky MA, Boothroyd DB, Bravata DM et al. Coronary artery bypass surgery compared with percutaneous coronary interventions for multivessel disease: a collaborative analysis of indivi- 
dual patient data from ten randomised trials. Lancet, 2009; 373: 1190-1197.

23. Flaherty JD, Davidson CJ. Diabetes and coronary revascularization. JAMA, 2005; 293: 1501-1508.

24. Kapur A, Hall RJ, Malik IS et al. Randomized comparison of percutaneous coronary intervention with coronary artery bypass grafting in diabetic patients. One-year results of the CARDia (Coronary Artery Revascularization in Diabetes) trial. J Am Coll Cardiol, 2010; 55: 432-440.

25. Mack MJ, Banning AP, Serruys PW et al. Bypass versus drug-eluting stents at three years in SYNTAX patients with diabetes mellitus or metabolic syndrome. Ann Thorac Surg, 2011; 92: 2140-2146.

26. Banning AP, Westaby S, Morice MC et al. Diabetic and nondiabetic patients with left main and/or 3-vessel coronary artery disease: comparison of outcomes with cardiac surgery and paclitaxel-eluting stents. J Am Coll Cardiol, 2010; 55: 1067-1075.

27. Sianos G, Morel MA, Kappetein AP et al. The SYNTAX Score: an angiographic tool grading the complexity of coronary artery disease. EuroIntervention, 2005; 1: 219-227.

28. Hannan EL, Wu C, Walford G et al. Drug-eluting stents vs. coronary-artery bypass grafting in multivessel coronary disease. N Engl J Med, 2008; 358: 331-341.

29. Przybysz-Zdunek B, Ploch M, Pluta W, Dada M, Opolski G. All cause readmission and repeat revascularization after percutaneous coronary intervention. Cardiol J, 2012; 19: 174-179.

30. Hlatky MA. Compelling Evidence for Coronary-Bypass Surgery in Patients with Diabetes. N Engl J Med, 2012 [Epub ahead of print].

31. Windecker S, Serruys PW, Wandel S et al. Biolimus-eluting stent with biodegradable polymer versus sirolimus-eluting stent with durable polymer for coronary revascularisation (LEADERS): A randomised non-inferiority trial. Lancet, 2008; 372: 1163-1173.

32. Kedhi E, Gomes ME, Lagerqvist B et al. Clinical impact of second-generation everolimus-eluting stent compared with first-generation drug-eluting stents in diabetes mellitus patients: Insights from a nationwide coronary intervention register. J Am Coll Cardiol Cardiovasc Interv, 2012; 5: 1141-1149.
33. Tzifos V, Gatsis A, Gerasimou A, Chatzis D, Theodorakis G. Acute and long term results of unprotected left main stenting using drug eluting stents. Cardiol J, 2011; 18: 165-170.

34. Grube E, Chevalier B, Guagliumi G et al. The SPIRIT V diabetic study: A randomized clinical evaluation of the XIENCE V everolimus-eluting stent vs. the TAXUS Liberté paclitaxel-eluting stent in diabetic patients with de novo coronary artery lesions. Am Heart J, 2012; 163: 867-875.

35. Kereiakes DJ, Sudhir K, Hermiller JB et al. Comparison of everolimus-eluting and paclitaxel-eluting coronary stents in patients undergoing multilesion and multivessel intervention: the SPIRIT III (A Clinical Evaluation of the Investigational Device XIENCE V Everolimus Eluting Coronary Stent System [EECSS] in the Treatment of Subjects With De Novo Native Coronary Artery Lesions) and SPIRIT IV (Clinical Evaluation of the XIENCE V Everolimus Eluting Coronary Stent System in the Treatment of Subjects With De Novo Native Coronary Artery Lesions) randomized trials. J Am Coll Cardiol Cardiovasc Interv, 2010; 3: 1229-1239.

36. Sheehy A, Hsu S, Bouchard A et al. Comparative vascular responses three months after paclitaxel and everolimus-eluting stent implantation in streptozotocin-induced diabetic porcine coronary arteries. Cardiovasc Diabetol, 2012; 11: 75.

37. Sedlis SP, Morrison DA, Lorin JD et al. Percutaneous coronary intervention versus coronary bypass graft surgery for diabetic patients with unstable angina and risk factors for adverse outcomes with bypass: Outcome of diabetic patients in the AWESOME randomized trial and registry. J Am Coll Cardiol, 2002; 40: 1555-1566 .

38. Yan $\mathrm{Q}$, Changsheng $\mathrm{M}$, Shaoping $\mathrm{N}$ et al. Percutaneous treatment with drug-eluting stent vs. bypass surgery in patients suffering from chronic stable angina with multivessel disease involving significant proximal stenosis in left anterior descending artery. Circ J, 2009; 73: 1848-1855.

39. Green L. Technoculture: From Alphabet to Cybersex. Allen \& Unwin, Sydney, Australia 2002.

40. Garg S, Serruys PW. An update on drug-eluting stents. Curr Treat Options Cardiovasc Med, 2012 [Epub ahead of print]. 